\title{
Early Predictive Value of Procalcitonin for the Diagnosis of Pulmonary Infections after Off-pump Coronary Artery Bypass Grafting
}

\author{
Yuntao Li $^{1}$, Jing Zhang ${ }^{2}$, ZiYang $\mathrm{He}^{2}$ \\ ${ }^{1}$ Department of Cardiac Surgery,Peking University International Hospital, Beijing ,China; \\ ${ }^{2}$ Department of Adult Surgical Intensive Care Unit, Fuwai Hospital, Beijing, China
}

\section{ABSTRACT}

Background: Cardiac surgery can cause similar inflammatory reactions with infection; antibacterial treatment may be inappropriately used. Early and accurate diagnosis of infection still is a difficult problem worldwide. Procalcitonin (PCT) helps to identify sepsis caused by bacterial infections. However, its application in the diagnosis of pulmonary infections after off-pump coronary artery bypass grafting (OPCABG) has not been well studied. We investigated the early predictive value of PCT for the diagnosis of pulmonary infections after OPCABG.

Methods: We retrospectively analyzed the clinical data, including conditions in the intensive care unit, postoperative complications, mortality rate, plasma PCT in the morning on the first postoperative day, routine white blood cell (WBC) count, and high-sensitivity C-reactive protein (hs-CRP) levels of patients who underwent elective OPCABG. Patients were divided into an infection group and a noninfection group, according to the occurrence of pulmonary infections. A receiver operating characteristic (ROC) curve was used to analyze the predictive value of PCT for the diagnosis of postsurgical infections.

Results: In total, 131 patients who underwent OPCABG were included, of whom $23(17.6 \%)$ developed pulmonary infections. The plasma PCT level significantly was higher in the infection group than in the noninfection group $(6.0 \pm$ $6.3 \mathrm{ng} / \mathrm{ml}$ vs. $2.0 \pm 2.2 \mathrm{ng} / \mathrm{ml}, P=0.007)$. WBC and hs-CRP values were not significantly different between the infection group and the noninfection group $(12.3 \pm 3.9 \times 109 / \mathrm{L}$ vs. 11.1 $\pm 2.8 \times 109 / \mathrm{L}, P=0.171$ and $12.4 \pm 0.7 \mathrm{mg} / \mathrm{L}$ vs. $12.4 \pm 0.8$ $\mathrm{mg} / \mathrm{L}, P=0.903$, respectively). The area under the ROC for predicting pulmonary infections after OPCABG by plasma PCT was $0.783(P<0.001$, with a $95 \%$ confidence interval of $0.674-0.893)$, with a cut-off value of $3.55 \mathrm{ng} / \mathrm{ml}$, a sensitivity of 0.609 , and a specificity of 0.861 .

Conclusion: From our study results, we postulate that PCT has a high early predictive value for the diagnosis of pulmonary infections after OPCABG.

Received October 12, 2020; accepted November 10, 2020

Correspondence: Yuntao Li, Peking University International Hospital, Beijing, China (e-mail: lyt19203@163.com)

\section{INTRODUCTION}

Antibiotic resistance has become a global concern, with the prevalence of serious infections rising every year. It is estimated that by 2050 , infectious diseases will cause 10 million deaths worldwide per year [Bartoletti 2018]. Infections can cause systemic inflammatory response syndrome (SIRS). In addition, surgical wounds and stress reactions can induce similar conditions [Wacker 2013], leading to difficulty in diagnosing postoperative infections. This can result in higher misdiagnosis rates of infections and increased use of antibiotics. Further dependence on infection sources and bacterial culture for diagnosis can delay accurate diagnoses or lead to false-negatives, and delayed anti-infective therapy may increase mortality rate [Gaieski 2010; Ferrer 2014]. Thus, early detection and diagnosis of postoperative infections remains a clinical concern.

Procalcitonin (PCT) can be used to differentiate between bacterial and nonbacterial sepsis. As suggested by several studies, PCT can be used as a biomarker for bacterial infections with high sensitivity and specificity [Wacker 2013, Prkno 2013]. Reports also have shown that the increased PCT can occur in noninfectious SIRS, such as multiple trauma and cerebral trauma, and after cardiac surgery, where PCT levels are related to the type of trauma and the severity of the condition [Wacker 2013, Uzzan 2006]. Various studies have been conducted on procalcitonin in the Department of Cardiac Surgery among patients who underwent on-pump cardiac surgeries [Aouifi 2000; Dorge 2003]. However, few data are available on the predictive value of PCT for the diagnosis of pulmonary infections after off-pump coronary artery bypass grafting (OPCABG).

Therefore, we investigated the early predictive value of PCT for diagnosing pulmonary infections in patients who underwent OPCABG.

\section{MATERIALS AND METHODS}

Patient selection: Adult patients ( $\geq 18$ years old) who underwent OPCABG in the north building of Fuwai Hospital, Beijing, China retrospectively were analyzed. Patients who had emergency surgeries, acute myocardial infarctions (within one month), other types infections (except pulmonary infection), or preoperative infections were excluded.

Postsurgical monitoring of procalcitonin: Following OPCABG, serum PCT of patients was recorded on the first 
Table 1. The clinical data of the infection and noninfection groups

\begin{tabular}{lccc}
\hline & Infection & Noninfection & $P$ \\
\hline Male & $18 / 23$ & $80 / 108$ & 0.675 \\
Age (year) & $64.6 \pm 8.9$ & $60.4 \pm 7.9$ & 0.025 \\
Height (cm) & $164.4 \pm 6.5$ & $168.8 \pm 7.6$ & 0.011 \\
Body weight (kg) & $67.1 \pm 9.2$ & $75 \pm 11.2$ & 0.002 \\
BMI (kg/m²) & $24.8 \pm 2.8$ & $26.2 \pm 2.9$ & 0.028 \\
LVEF (\%) & $58 \pm 9.5$ & $59.1 \pm 8.2$ & 0.549 \\
Hypertension & $18 / 23$ & $76 / 108$ & 0.445 \\
Type-2 DM & $11 / 23$ & $56 / 108$ & 0.726 \\
Prior cerebral infraction & $4 / 23$ & $7 / 101$ & 0.102 \\
Preoperative WBC (10^9/L) & $6.9 \pm 1.7$ & $6.0 \pm 1.4$ & 0.008 \\
Postoperative WBC (10^9/L) & $12.3 \pm 3.9$ & $11.1 \pm 2.8$ & 0.171 \\
Mechanical ventilation time (hrs) & $29 \pm 18.7$ & $20 \pm 11.6$ & 0.036 \\
Length of ICU (hrs) & $96.3 \pm 91.1$ & $46.1 \pm 33.7$ & 0.016 \\
Hs-CRP (mg/L) & $12.4 \pm 0.7$ & $2.4 \pm 0.8$ & 0.903 \\
PCT (ng/ml) & $6.0 \pm 6.3$ & $2.0 \pm 2.2$ & 0.007 \\
MACE & $2 / 23$ & $2 / 106$ & 0.142 \\
\hline
\end{tabular}

WBC: white blood cell; LVEF: left ventricular ejection fraction; Hs-CRP: highsensitivity C-reactive protein; PCT: procalcitonin; MACE: main adverse cardiac events, including peri-operative myocardial infarction, death, and stroke

postoperative morning and routinely thereafter. Simultaneously, we recorded patients' demographic characteristics; preoperative comorbidities; preoperative and postoperative inflammatory markers; duration of mechanical ventilation; length of ICU stay; and occurrence of major adverse cardiac events (MACE), including stroke, myocardial infarction, and death. (Table 1)
According to the diagnostic criteria published by the Centers for Disease Control and Prevention/National Healthcare Safety Network (CDC/NHSN) [Horan 2008], patients were divided into an infection group and a noninfection group. (Table 2)

Statistical methods: In this study, continuous variables were presented as means \pm standard deviations and categorical variables as percentages. To compare data between the two groups, an independent-sample t-test was used for continuous variables, whereas the chi-squared test or Fisher's exact test was used for categorical variables. A receiver operating characteristic (ROC) curve was used to determine the diagnostic test efficiency and critical values. Differences were considered statistically significant at $P<0.05$. All data were statistically analyzed using SPSS 25.0.

\section{RESULTS}

General information: Overall, 131 patients (98 men and 33 women; mean age of $61.1 \pm 8.2$ years) who received OPCABG were included. Of them, 23 (17.6\%) had pulmonary infections. Patients in the infection group were older and had a lower body weight and higher preoperative WBC level than those in the noninfection group (Table 1).

Inflammatory markers: Patients in the infection group had significantly higher plasma PCT levels than those in the noninfection group $(6.0 \pm 6.3$ vs. $2.0 \pm 2.2, P=0.007)$ on the first postoperative day. The postoperative $\mathrm{WBC}$ and CRP levels were not significantly different between the infection group and the noninfection group $(12.3 \pm 3.9 \times 109 / \mathrm{L}$ vs. $11.1 \pm 2.8 \times 109 / \mathrm{L}, P=0.171$, and $12.4 \pm 0.7 \mathrm{mg} / \mathrm{L}$ vs. $12.4 \pm 0.8$ $\mathrm{mg} / \mathrm{L}, P=0.903$, respectively). Moreover, the infection group had significantly higher preoperative WBC levels than the noninfection group $(6.9 \pm 1.7$ vs. $6.0 \pm 1.4, P=0.008)$. (Figure 1 )

Predictive value of plasma PCT for infections: The area under the curve (AUC) of ROC of the predictive value

Table 2. Algorithms for clinically defined pneumonia

\begin{tabular}{|c|c|}
\hline Two or more serial chest radiographs with at least one of the following: & FOR ANY PATIENT, at least one of the following: \\
\hline Consolidation & Leukopenia $\left(<4000 \mathrm{WBC} / \mathrm{mm}^{3}\right)$ or leukocytosis $\left(\geq 12,000 \mathrm{WBC} / \mathrm{mm}^{3}\right)$ \\
\hline Cavitation & For adults $\geq 70$ years old, altered mental status with no other recognized cause \\
\hline & and at least two of the following: \\
\hline & $\begin{array}{l}\text { New onset of purulent sputum or change in character of sputum or in- } \\
\text { creased respiratory secretions or increased suctioning requirements }\end{array}$ \\
\hline & New onset or worsening cough, or dyspnea, or tachypnea \\
\hline & Rales or bronchial breath sounds \\
\hline
\end{tabular}


of plasma PCT for pulmonary infections after OPCABG was $0.783(P<0.001)$, with a $95 \%$ confidence interval of $0.674-$ 0.893 , a cut-off value of $3.55 \mathrm{ng} / \mathrm{ml}$, a sensitivity of 0.609 , and a specificity of 0.861 . (Figure 2 )

Analyses of prognostic indicators: The infection group had a significantly prolonged mechanical ventilation and length of ICU stay $(29.0 \pm 18.7 \mathrm{~h}$ vs. $20 \pm 11.6 \mathrm{~h}, P=0.036$, and $96.3 \pm 91.1$ h vs. $46.1 \pm 33.7 \mathrm{~h}, P=0.016$, respectively) compared with the noninfection group. In the infection group, four patients had MACE; among them, three had perioperative myocardial infarction (one died of this adverse event), and one had stroke. The infection group had a higher incidence rate of MACE; however, the incidence rates of MACE were not significantly different between the two groups $(8.7 \%$ vs. $1.9 \%, P=0.142)$.

\section{DISCUSSION}

Due to contributors, such as inappropriate antibiotic use or abuse, antibiotic resistance has become a severe concern globally. Even now, 30-40\% of antibiotic prescriptions come with inappropriate indications or inappropriate doses, times, or durations [Huttner 2013; Ventola 2015]. Infection is easy to diagnose if sterile body fluid culture is positive and infection symptoms are present. However, bacterial cultures need a long time, and the positivity rate is low. Furthermore, infections and trauma can cause similar inflammatory reactions, such as fever, increased WBC and CRP; therefore, antibacterial treatment may be delayed or inappropriately used, and these factors can all lead to increased mortality. Early and accurate diagnosis of infection is still a difficult problem worldwide. If a biomarker could distinguish infection and trauma, it would be of great significance.

PCT is a precursor of calcitonin present in various organs and tissues [Muller 2001]. Studies have shown that PCT can be an inflammatory marker for bacteria-induced sepsis because its synthesis increases during the acute phase of infections [Assicot 1993]. PCT also increases rapidly after infection and is related to the severity of inflammatory responses [Dandona 1994]. PCT has a short half-life and decreases spontaneously in the absence of appropriate stimuli [Assicot 1993; Kallel 2012]. Therefore, PCT has high sensitivity and specificity as a biomarker for bacterial infections [Wacker 2013, Prkno 2013].

After thoracotomy, due to factors, such as pain, decreased lung and chest wall compliance, and difficulty in expectorating sputum, patients are likely to have reduced respiratory function, which may lead to pulmonary atelectasis and pulmonary infections [Wynne 2004]. In addition, because noninfectious factors, such as surgical wounds, may lead to systemic inflammatory responses, the diagnosis of postthoracotomy pulmonary infection is challenging, which can lead to higher diagnosis rates of pulmonary infections and increased use of antibiotics. Antibiotic overuse is related to increased treatment cost, adverse events, and prolonged hospital stays.

An expert consensus on PCT-guided antibiotic therapy published in 2018 for patients in the ICU suggested that [Bartoletti 2018] 1) PCT should be used to guide anti-infective

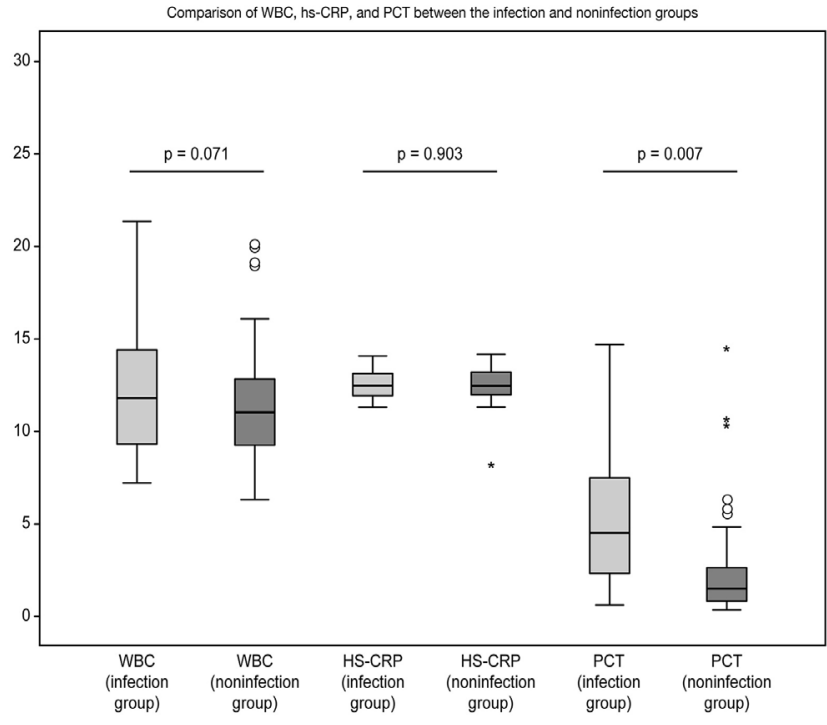

Figure 1. Comparison of WBC, hs-CRP, and PCT between the infection and noninfection groups. The infection group had significantly higher PCT levels than the noninfection group $(6.0 \pm 6.3 \mathrm{ng} / \mathrm{ml}$ vs. $2.0 \pm 2.2 \mathrm{ng} / \mathrm{ml}$, $P=0.007)$. WBC count and hs-CRP level were not significantly different between the two groups $(12.3 \pm 3.9 \times 109 / \mathrm{L}$ vs. $11.1 \pm 2.8 \times 109 / \mathrm{L}, P=$ 0.171 and $12.4 \pm 0.7 \mathrm{mg} / \mathrm{L}$ vs. $12.4 \pm 0.8 \mathrm{mg} / \mathrm{L}, P=0.903$, respectively).

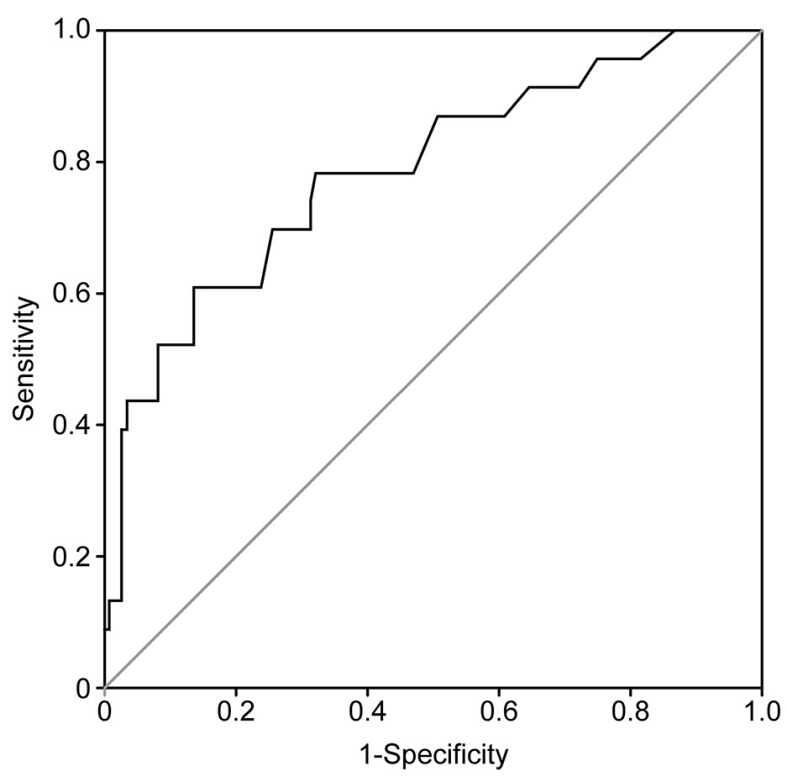

Figure 2. The ROC curve of the predictive value of PCT for pulmonary infections after OPCABG. The area under the curve (AUC) of ROC was $0.783(P<0.001)$, with a $95 \%$ confidence interval of $0.674-0.893$, cut-off value of $3.55 \mathrm{ng} / \mathrm{ml}$, sensitivity of 0.609 , and specificity of 0.861 .

treatments, and 2) in the ICU, PCT must be included in the anti-infective treatment framework, and dynamic PCT changes should be monitored to guide the discontinuation of antibiotics. Despite numerous studies on PCT, no previous 
study has provided a unified specification for the application of PCT under specific clinical situations, although its critical value for diagnosing infections can vary clinically.

In a meta-analysis that included 3,233 critical patients with sepsis in 30 studies, the critical value of PCT varied greatly, ranging from 0.1-15.75 ng/ml [Wacker 2013]. Wang et al. [Wang 2017] showed that, for critical patients who developed SIRS after on-pump cardiac surgery, the cut-off value of PCT for the diagnosis of pulmonary infections on the first postoperative day was $6.8 \mathrm{ng} / \mathrm{ml}$. Because OPCABG avoids extracorporeal circulation for patients and leads to less severe inflammatory responses, the cut-off values of PCT for diagnosing pulmonary infections might be different after on-pump and off-pump cardiac surgeries. Therefore, in our study, the cut-off value of PCT for the diagnosis of pulmonary infection was 3.55 $\mathrm{ng} / \mathrm{ml}$, and the AUC of ROC was $0.783(P<0.001$, with a $95 \%$ confidence interval of 0.674-0.893), suggesting that PCT had a high diagnostic value for postoperative pulmonary infections.

Plasma PCT levels were significantly increased in patients who developed pulmonary infections after OPCABG, whereas the levels of inflammatory response markers-WBC and CRP-were not significantly different between the two groups. Elevated plasma PCT is closely related to pulmonary infection; thus, PCT is a better indicator for postoperative infections than WBC and CRP. This is consistent with the results of other studies that indicated a positive correlation between the elevation of PCT levels and disease severity in patients with sepsis. This showed that PCT had a higher specificity and sensitivity than inflammation markers, such as CRP, WBC, and the percentage of neutrophils [Zhao 2015]. A plasma PCT level higher than the cut-off value is helpful in the early prediction of postoperative infections and is of high reference value for guiding anti-infective treatments, developing anti-infection strategies, and improving clinical prognosis.

We also found that some patients had pulmonary infection with low procalcitonin levels, while other patients had high procalcitonin levels without pulmonary infection. This could be attributed to two possible reasons.

First, in this study, we only analyzed the relationship between procalcitonin and infection on the first morning after the operation. We could not predict whether there were other iatrogenic factors causing pulmonary infection in the follow-up treatment, such as sputum aspiration, fiberoptic bronchoscopy operation, aspiration, and delayed extubation. Therefore, our aim was to observe the procalcitonin levels in this study. If the trend showed an increase, it indicated a high possibility of infection.

Second, surgical trauma also could have caused inflammation and increased the procalcitonin levels. We examined the differences in the procalcitonin levels among patients to observe their individual responses to trauma.

In conclusion, trauma can cause inflammatory reactions similar to infections, which may lead to an increase in misdiagnosis rates of postoperative infection and antibiotic use. Compared with other inflammatory markers, PCT level was significantly elevated in pulmonary infection patients. Plasma PCT level has a high early predictive value for pulmonary infections after OPCABG. This conclusion still requires large-scale studies for confirmation.

\section{REFERENCES}

Aouifi A, Piriou V, Bastien O, Blanc P, Bouvier H, Evans R, Celard M, Vandenesch F, Rousson R, Lehot JJ. 2000. Usefulness of procalcitonin for diagnosis of infection in cardiac surgical patients. Crit Care Med. 28(9):3171-3176.

Assicot M, Gendrel D, Carsin H, Raymond J, Guilbaud J, Bohuon C. 1993. High serum procalcitonin concentrations in patients with sepsis and infection. Lancet. 341(8844):515-518.

Bartoletti M, Antonelli M, Bruno Blasi FA, Casagranda I, Chieregato A, Fumagalli R, Girardis M, Pieralli F, Plebani M, Rossolini GM, et al. 2018. Procalcitonin-guided antibiotic therapy: An expert consensus. Clin Chem Lab Med 56(8):1223-1229.

Dandona P, Nix D, Wilson MF, Aljada A, Love J, Assicot M, Bohuon C. 1994. Procalcitonin increase after endotoxin injection in normal subjects. J Clin Endocrinol Metab. 79(6):1605-1608.

Dorge H, Schondube FA, Dorge P, Seipelt R, Voss M, Messmer BJ. 2003. Procalcitonin is a valuable prognostic marker in cardiac surgery but not specific for infection. Thorac Cardiovasc Surg. 51(6):322-326.

Ferrer R, Martin-Loeches I, Phillips G, Osborn TM, Townsend S, Dellinger RP, Artigas A, Schorr C, Levy MM. 2014. Empiric antibiotic treatment reduces mortality in severe sepsis and septic shock from the first hour: Results from a guideline-based performance improvement program. Crit Care Med. 42(8):1749-1755.

Gaieski DF, Mikkelsen ME, Band RA, Pines JM, Massone R, Furia FF, Shofer FS, Goyal M. 2010. Impact of time to antibiotics on survival in patients with severe sepsis or septic shock in whom early goal-directed therapy was initiated in the emergency department. Crit Care Med. 38(4):1045-1053.

Horan TC, Andrus M, Dudeck MA. 2008. CDC/NHSN surveillance definition of health care-associated infection and criteria for specific types of infections in the acute care setting. Am J Infect Control. 36(5):309-332.

Huttner A, Harbarth S, Carlet J, Cosgrove S, Goossens H, Holmes A, Jarlier V, Voss A, Pittet D. 2013. Antimicrobial resistance: A global view from the 2013 World Healthcare-Associated Infections Forum. Antimicrob Resist Infect Control. 2:31.

Kallel S, Abid M, Jarraya A, Abdenadher M, Mnif E, Frikha I, Ayadi F, Karoui A. 2012. Kinetics, diagnostic and prognostic value of procalcitonin after cardiac surgery. Ann Biol Clin. 70(5):567-580.

Muller B, White JC, Nylen ES, Snider RH, Becker KL, Habener JF. 2001. Ubiquitous expression of the calcitonin-i gene in multiple tissues in response to sepsis. J Clin Endocrinol Metab. 86(1):396-404.

Prkno A, Wacker C, Brunkhorst FM, Schlattmann P. 2013. Procalcitoninguided therapy in intensive care unit patients with severe sepsis and septic shock: A systematic review and meta-analysis. Crit Care. 17(6):R291.

Uzzan B, Cohen R, Nicolas P, Cucherat M, Perret GY. 2006. Procalcitonin as a diagnostic test for sepsis in critically ill adults and after surgery or trauma: A systematic review and meta-analysis. Crit Care Med. 34(7):1996-2003.

Ventola CL. 2015. The antibiotic resistance crisis: Part 1: Causes and threats. P T. 40(4):277-283.

Wacker C, Prkno A, Brunkhorst FM, Schlattmann P. 2013. Procalcitonin as a diagnostic marker for sepsis: A systematic review and meta-analysis. Lancet Infect Dis. 13(5):426-435.

Wang H, Cui N, Niu F, Xu H, Long Y, Liu D. 2017. Usefulness of procalcitonin for the diagnosis of infection in cardiac surgical patients. 29(10):897-901. 
Wynne R, Botti M. 2004. Postoperative pulmonary dysfunction in adults after cardiac surgery with cardiopulmonary bypass: Clinical significance and implications for practice. Am J Crit Care. 13(5):384-393.
Zhao L, Zang X, Chen W, Sheng B, Gu X, Zhang J. 2015. Analysis of correlation between inflammatory parameters and severity of sepsis caused by bacterial bloodstream infection in septic patients. 27(6):448-453. 